\title{
Author Correction: Synergetic coordination and catecholamine chemistry for catalytic generation of nitric oxide on vascular stents
}

Xiangyang Li, Hua Qiu, Peng Gao, Ying Yang, Zhilu Yang and Nan Huang

\section{Correction to: NPG Asia Materials}

https://doi.org/10.1038/s41427-018-0052-3

published online 06 June 2018

The published version of this article contains errors in Fig. 5a, Fig. 6a and Fig. 7a.

1. In the donor (-) group of Fig. 5a the SEM images of platelets on the samples of 12.5 and 50 are the same. However, this mistake would not cause comprehension difficulty and affect the present conclusions. Because there were no NO releasing between these two groups without the supplement of donor. Thus the adhesion and spreading of platelets on these samples were almost the same.

2. In the donor $(+)$ group of Fig. 6a, the fluorescence staining of HUASMCs on the samples of 25 and 50 are the same. However, this mistake would not cause comprehension difficulty and affect the present conclusions. The result of CCK-8 (Fig. 6c) showed no significant difference of cell viability for these two groups. Moreover, as we reported recently ${ }^{1}$, the surface releasing $\mathrm{NO}$ with a rate above $4 \times 10^{-10}$ mol cm${ }^{-2} \mathrm{~min}^{-1}$ show little difference of inhibition efficiency of HUSMCs. The catalytic generating NO rate of both sample of 25 and 50 are above $4 \times 10^{-10}$ mol cm${ }^{-2} \min ^{-1}$ in this study.

3. In the donor $(-)$ group of Fig. 7a, the rhodamine staining images of HUVECs $(2 \mathrm{~h})$ on the samples of 3.125 and 6.25 are the same, as well as the 12.5 and 25. However, this mistake would not cause comprehension difficulty and affect the present conclusions, because there were no NO releasing for these groups, thus it has little difference on adhesion and proliferation of HUVECs between these groups.

Considering all the mistakes will not affect the conclusion of this study, we want to replace the wrong images with the correct ones that provide below but would not do any change to the text.

The original article has been corrected.

The authors apologize for these errors!

Published online: 15 March 2021

\section{Reference}

1. Gao, P. et al. Metal-catechol-(amine) networks for surface synergistic catalytic modification: therapeutic gas generation and biomolecule grafting. Biomaterials 248, 119981 (2020). 


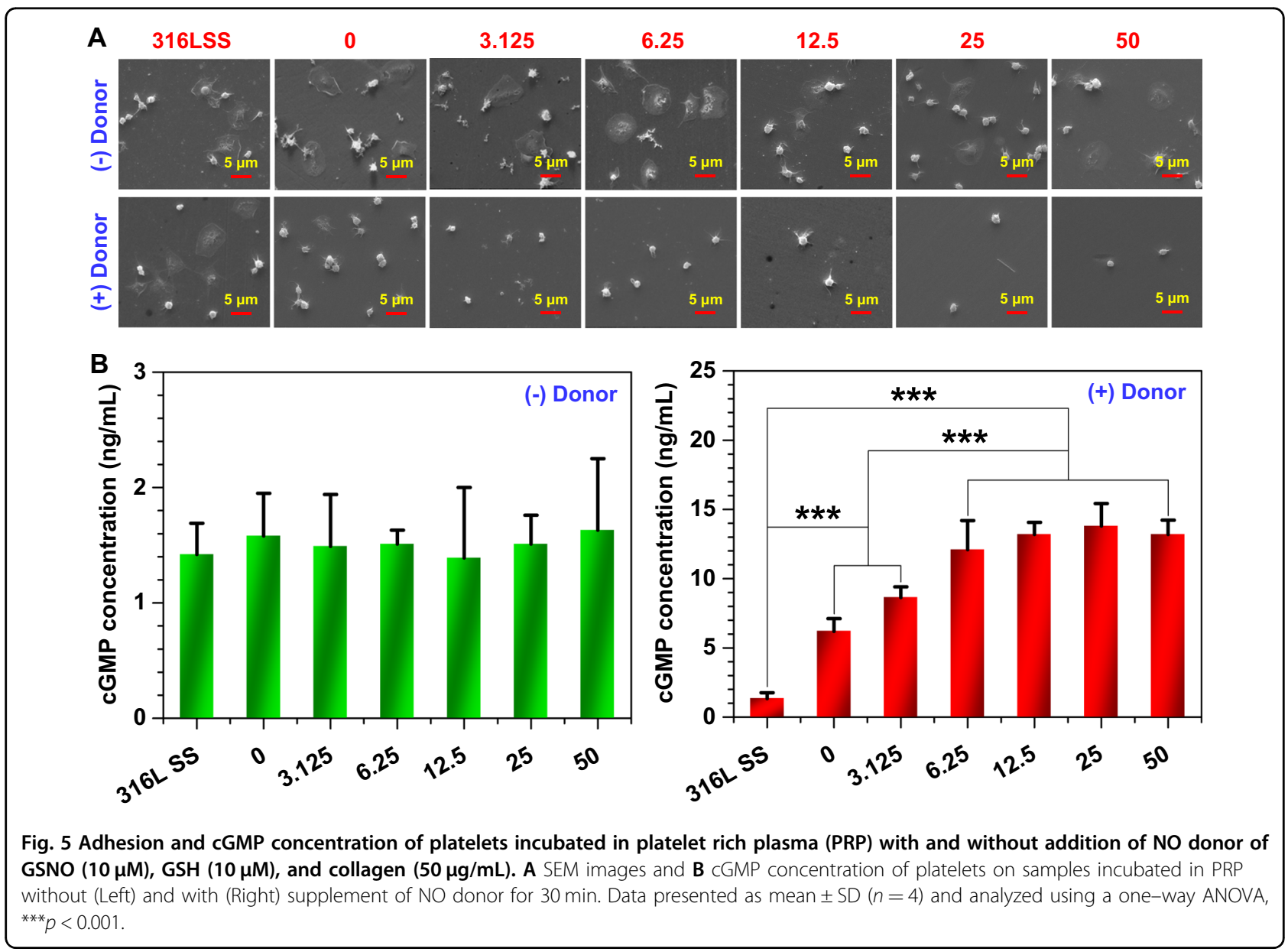




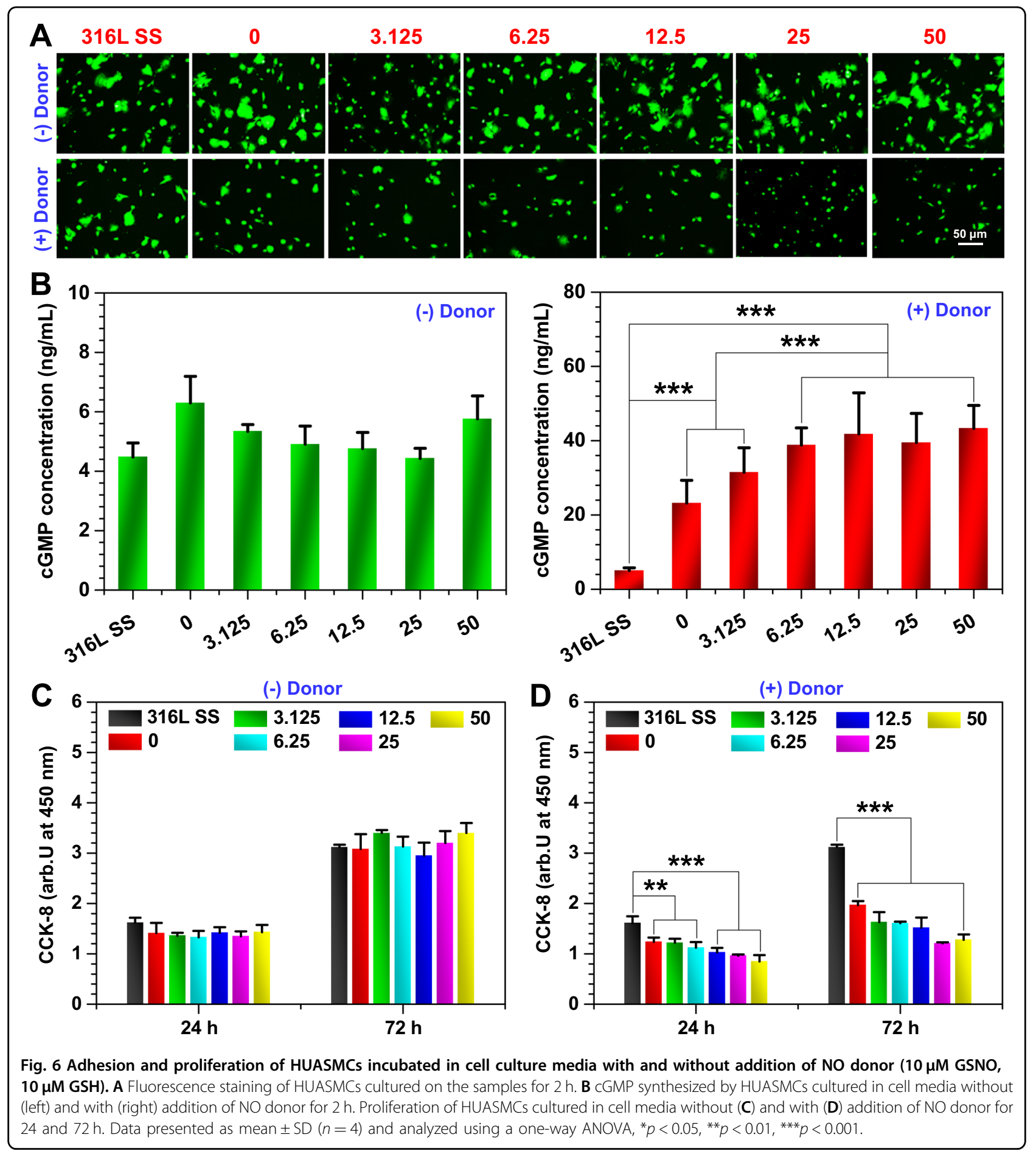




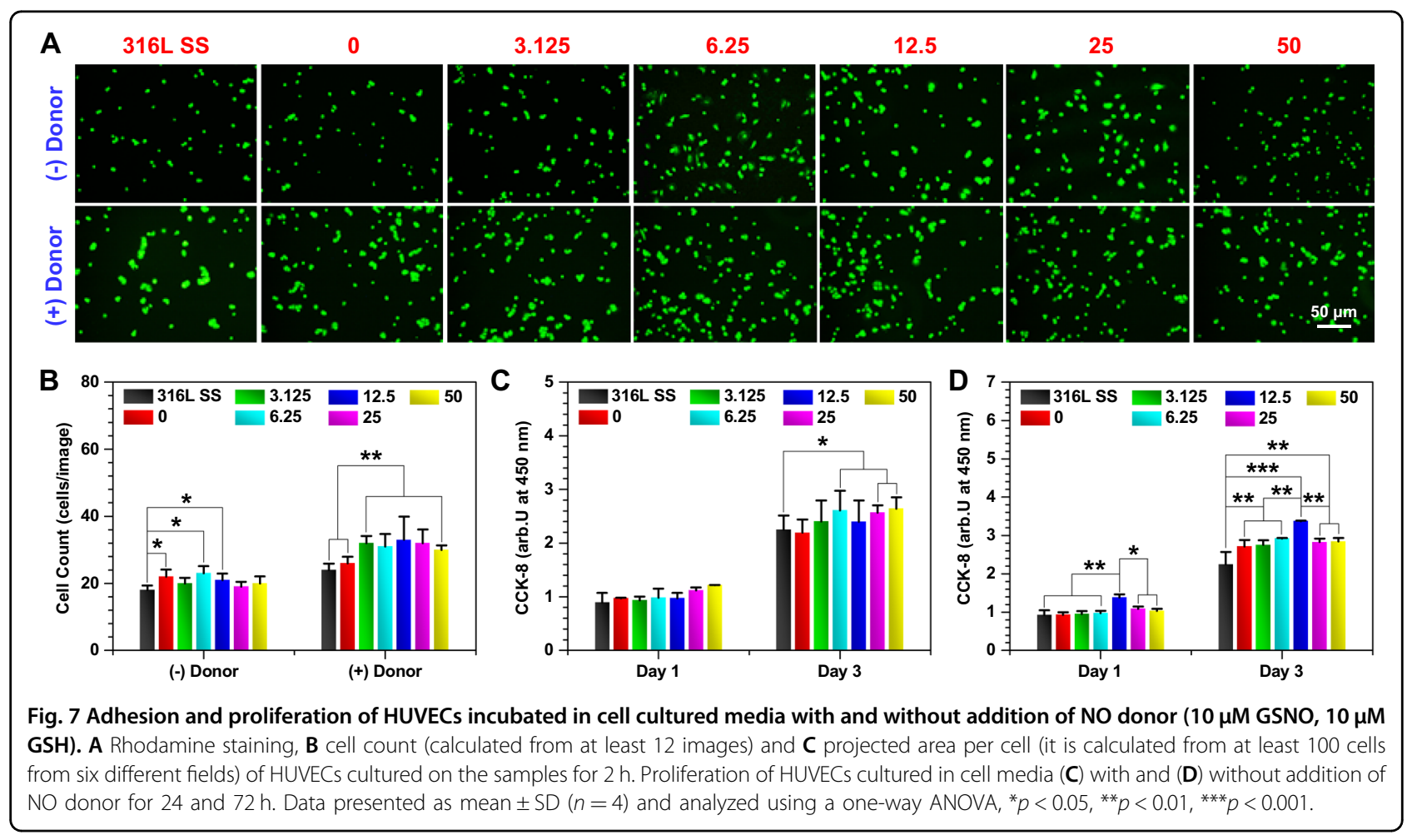

